\title{
Modulation by Centrifugation of Cell Susceptibility to Chlamydial Infection
}

\author{
By I. ALLAN AND J. H. PEARCE \\ Department of Microbiology, University of Birmingham, B15 2TT
}

(Received 31 July 1978)

\begin{abstract}
Enhancement of chlamydial infection of cell monolayers by centrifugation was shown to depend on induced cell surface changes. Evidence for this came from analysis of two forms of organism attachment which take place during centrifugation. In 'productive binding', organisms attached to cells and then entered and infected them. In 'unproductive binding', organisms became attached to cells but were not ingested. These organisms could be stripped from the cells by treatment with trypsin and could then infect fresh monolayers.

Measurement of attachment kinetics during centrifugation showed that cells passed through three different susceptibility states. Only productive binding occurred in the first $20 \mathrm{~min}$; cells then entered a refractory state during which no attachment took place. At about $45 \mathrm{~min}$, attachment recommenced but this allowed only unproductive binding. Induced movement of cell surface structures may enhance infection by promoting specific or non-specific interactions. Failure of ingestion may result from insufficient cell 'receptors' for circumferential binding of the whole chlamydial surface so that engulfment cannot take place.
\end{abstract}

\section{INTRODUCTION}

Mechanisms of particle endocytosis by 'professional' and 'non-professional' or 'facultative' phagocytes (Silverstein et al., 1977) are of considerable importance in relation to microbial penetration of epithelial surfaces. A special case of this general phenomenon is the greatly increased attachment and enhanced infection resulting from centrifugation, at 1000 to $2000 \mathrm{~g}$, of viruses or chlamydiae with cell monolayers. The enhancement has been attributed to increased collisions resulting from sedimentation (Osborn \& Walker, 1968), but it is now clear that, at the low centrifugal forces shown to be effective, sedimentation cannot be a significant factor for the smaller viruses (Bryden et al., 1977).

Using Chlamydia psittaci, strain 'guinea pig inclusion conjunctivitis' (GP-IC), we have previously shown that attachment and infection are not invariable consequences of centrifugation but are also dependent on cell surface properties which can be modulated by cell growth in different sera (Allan \& Pearce, 1977). Three observations indicate that organismcell surface interactions during centrifugation may differ from those in spontaneous (static cell) infection. First, antibody can block spontaneous infection but the inhibition is overcome by centrifugation (Ainsworth et al., 1976; Allan et al., 1977). Second, at $4{ }^{\circ} \mathrm{C}$ attachment is greatly reduced in centrifuge-assisted infection (Griffiths et al., 1976) but unimpaired in spontaneous infection (unpublished). Third, there is a correlation between the increased pressure generated during centrifuging and the enhancement of infection. Maximum infection could be achieved on centrifugation at $69 \mathrm{~g}$ by layering immiscible fluid above the inoculum to act as a fluid piston during centrifuging (Allan et al., 1977). The low 
centrifugal force rules out sedimentation as a factor in chlamydial enhancement; the pressure effect would seem more likely to be expressed on the deformable cell surface than on the rigid-walled chlamydial particle suggesting that some form of centrifugation-induced cell response facilitates infection.

We present here evidence that centrifugation induces cell surface changes affecting susceptibility to infection and show that successive, transient cell states develop which affect both organism attachment and ingestion.

\section{METHODS}

Organism. The guinea pig inclusion conjunctivitis strain (GP-IC) of Chlamydia psittaci was grown in eggs and harvested as previously described (Griffiths et al., 1976). Organisms were purified using Renografin76 (E. R. Squibb and Sons, New York, U.S.A.) by the batch procedure of Howard et al. (1974).

Cell culture and monolayer preparation. McCoy cells were cultured in growth medium supplemented with $5 \%(\mathrm{v} / \mathrm{v})$ foetal bovine serum (FBS), irradiated, and monolayers were prepared on coverslips (Allan et al., 1977).

Infectivity titration on cell monolayers. In all experiments, irradiated cells were infected 4 or $5 \mathrm{~d}$ after seeding of monolayers. Strain GP-IC was inoculated on to monolayers at doses sufficient to infect up to $10 \%$ of cells and titrated for infectivity (Griffiths et al., 1976) by centrifuging with monolayers at $1580 \mathrm{~g}$ and $37^{\circ} \mathrm{C}$ for $60 \mathrm{~min}$, unless otherwise stated; 2500 cells from each of four replicate monolayers were examined microscopically for infected cells.

In certain experiments it was found that, after centrifugation, the number of infected cells in monolayers was increased by cold shock and recentrifugation. Briefly, after centrifugation and removal of supernatants, monolayers were cold-shocked by rinsing with diluent [Hanks' balanced salts solution (HBSS) with $5 \%$ (v/v) FBS] at $0^{\circ} \mathrm{C}$; diluent warmed to $37^{\circ} \mathrm{C}$ was then added and the monolayers were recentrifuged. Inoculated control monolayers, rinsed with diluent at $37^{\circ} \mathrm{C}$, showed no increase in titre after recentrifuging.

Titration of extracellular, cell-attached organism infectivity. Cell-attached organisms were detected after centrifuging the inoculum with monolayers. They were titrated as follows. Monolayers were treated for $5 \mathrm{~min}$ with trypsin [0.2 ml per rinsed coverslip, type XII trypsin (Sigma), at $\left.20 \mu \mathrm{g} \mathrm{ml}^{-1}\right]$ and then an equal volume of HBSS with $10 \%$ (v/v) FBS was added. The resulting suspension of detached cells and stripped organisms was centrifuged at $100 \mathrm{~g}$ for $5 \mathrm{~min}$. The supernatant, containing the stripped extracellular organisms, was titrated for infectivity by centrifugation with fresh monolayers. The cell pellet (containing intracellular infective organisms) did not show significant cell damage as judged by dye exclusion; the pellet was resuspended in Eagle's minimum essential medium with $5 \%$ (v/v) FBS, pipetted on to coverslips and, after reattachment of cells, incubated for inclusion development by intracellular organisms. About 80 to $90 \%$ of the cells treated with trypsin were recovered and reattached to coverslips; infectivity titres are corrected for this reduction.

Delayed inoculation of monolayers during centrifuging. In certain experiments the technique of 'delayed inoculation' was applied, by which organisms were introduced to monolayers at intervals after the centrifuge had been set in motion. For this purpose, the centrifuge was modified to allow the passage of inoculum through flexible plastic tubing carefully positioned at the centre of the rotor. The tubing ran out to a swinging bucket carrying a bottle with monolayer. At speed, the open end of the tubing at the centre of rotation was stationary in cross-section when viewed from above and the inoculum was readily introduced by hypodermic syringe. Control monolayers inoculated by this means immediately after the centrifuge had reached full speed gave infectivities identical with conventionally inoculated monolayers.

\section{RESULTS}

\section{Productive and unproductive binding}

During centrifuge-assisted infection strain GP-IC showed two kinds of attachment to cell monolayers. In the first, 'productive binding', organisms attached to cells and then entered and infected them; this gave the infectivity which is normally measured by the procedure (Table 1a). In the second, 'unproductive binding', organisms attached to cells but were not ingested. Their presence could be demonstrated by treating the monolayer with trypsin (which stripped off extracellular organisms) and titrating the trypsin supernatant on fresh monolayers [Table $1 b(i i)]$. When the trypsin-treated cells (which contained 


\section{Table 1. Demonstration of 'productive' and 'unproductively bound' organisms after centrifugation with monolayers}

Strain GP-IC inoculum was titrated for infectivity by centrifugation with monolayers of McCoy cells for $60 \mathrm{~min}$; other titres are expressed relative to this value. Monolayers that had been centrifuged with organisms at the same time were rinsed and treated with trypsin to remove extracellular (unproductively bound) organisms. The resulting suspension of detached cells and stripped organisms was centrifuged at $100 \mathrm{~g}$ for $5 \mathrm{~min}$. The trypsin supernatant (extracellular organisms) was titrated for infectivity on fresh monolayers. The cell pellet was resuspended, the cells were allowed to reattach to coverslips and incubated for development of inclusions from intracellular, productively bound organisms (titres are corrected for the small proportion of cells which failed to reattach).

Treatment of monolayers after addition of organisms

(a) Centrifugation

(b) Centrifugation, trypsin treatment (i) cells

(ii) trypsin supernatant on fresh monolayers
Relative infectivity titre

$1 \cdot 00$

0.93

Table 2. Entry and infection of monolayers by 'unproductively bound' organisms after cold shock and recentrifugation

Titres are expressed relative to the infectivity titre obtained after centrifugation of strain GP-IC with monolayers of McCoy cells for $60 \mathrm{~min}$. Following centrifuge-assisted infection and subsequent removal of supernatants, monolayers were treated with the combinations of cold shock, recentrifugation and trypsin shown below.

\section{Treatment of monolayers after addition of organisms}

(a) Centrifugation, cold shock, recentrifugation

(b) Centrifugation, cold shock, recentrifugation, trypsin treatment

(i) cells

(ii) trypsin supernatant on fresh monolayers

(c) Centrifugation, recentrifugation

(d) Centrifugation, recentrifugation, trypsin treatment (i) cells

(ii) trypsin supernatant on fresh monolayers

(e) Centrifugation, cold shock

(f) Centrifugation, cold shock, trypsin treatment

(i) cells

(ii) trypsin supernatant on fresh monolayers
Relative infectivity titre

$1 \cdot 92$

$1 \cdot 90$

0.03

1.02

$1 \cdot 06$

$0 \cdot 89$

1.09

$0 \cdot 98$

$1 \cdot 04$

intracellular, productively bound organisms) were reattached to coverslips, they gave the 'normal' infectivity titre [Table $1 b(i)]$.

Infectivity could be increased if monolayers were cold-shocked and recentrifuged after the initial centrifuge-assisted infection (Table 2a). Analysis showed that this procedure increased infectivity as a result of entry and infection of previously unproductively bound organisms. Thus, after cold shock and recentrifugation all infectivity was associated with the cells and none could be stripped by trypsin and released into supernatants [Table $2 b(i)$ and $b(i i)]$. Centrifugation and recentrifugation, without cold shock, did not increase infectivity and the extracellular organisms which remained could be removed by trypsin [Table $2 c, d(i)$ and $d(i i)$ ]. Similarly, centrifugation and cold shock, without recentrifugation, did not alter the infectivity distribution [Table $2 e, f(i)$ and $f(i i)$ ]. 


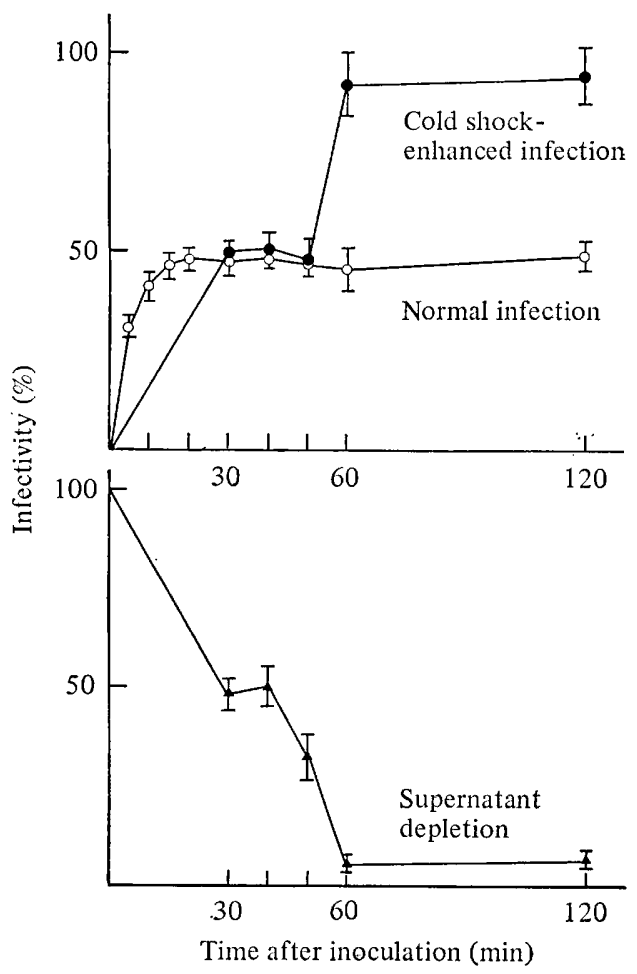

Fig. 1. Kinetics of normal infection, cold shock-enhanced infection and supernatant depletion. Monolayers of McCoy cells were centrifuged with similar inocula of strain GP-IC for various periods of time. For normal infection (productive binding only, $\mathrm{O}$ ), monolayers, after removal of supernatants, were incubated for development of inclusions. After centrifuging for between 30 and $120 \mathrm{~min}$, replicate monolayers were examined for cold shock-enhanced infection (productive and unproductive binding, -), after removal of supernatants, by cold shock and recentrifugation (see Methods); the supernatants were titrated for infectivity $(\boldsymbol{\Lambda})$ on fresh monolayers by centrifugation for $60 \mathrm{~min}$, cold shock and recentrifugation. All infectivities are expressed as a percentage of the inoculum infectivity summed from that detected at $60 \mathrm{~min}$ in cold shock-enhanced infection and that in the corresponding supernatant; results are the mean, \pm S.D., of four replicates.

\section{Kinetics of productive and unproductive binding}

Examination of the kinetics of these two forms of attachment provided clear evidence of cell surface changes during centrifugation. Monolayers were inoculated to produce 5 to $10 \%$ infected cells and sampled at intervals during centrifugation. Attachment was rapid during the first $20 \mathrm{~min}$ of centrifuging (Fig. 1, normal infection). Replicate monolayers taken at later times were, after removal of the supernatants, either incubated for development of infected cells arising from productive binding (Fig. 1, normal infection) or coldshocked, recentrifuged and incubated for development of infected cells arising from both productive and unproductive binding (Fig. 1, cold shock-enhanced infection). In parallel, the supernatants were titrated for residual infectivity on fresh monolayers, using cold shock enhancement to maximize infectivity detection. Comparison of the two infection curves shows that productive and unproductive binding took place sequentially and not simultaneously. Thus, over the first 30 min binding was solely productive. By this time cells appeared to have entered into a refractory state during which no attachment occurred although many organisms were present in the supernatants. At about 45 min, further attachment took place indicating the appearance of a new cell state. This latter was evidently different from the initial state developing after the onset of centrifugation since wholly unproductive binding occurred (Fig. 1). 


\section{Table 3. Independence of developing cell insusceptibility from presence of inoculum and its reversible nature}

In treatments $(a, c, d, e)$, monolayers were inoculated and centrifuged as before; the rest periods between centrifugation runs were approx. $2 \mathrm{~min}$. In treatment $(b)$, organisms were introduced to monolayers $20 \mathrm{~min}$ after the centrifuge had reached full speed (see Methods).

\section{Treatment of monolayers}

(a) Centrifugation with inoculum for $20 \mathrm{~min}$

(b) Centrifugation for $20 \mathrm{~min}$, inoculum added during centrifuging and present from 20 to $60 \mathrm{~min}$

(c) Centrifugation with inoculum for $60 \mathrm{~min}$

(d) Centrifugation for $60 \mathrm{~min}$, inoculum added during rest, further centrifugation for $60 \mathrm{~min}$

(e) Centrifugation with inoculum for $20 \mathrm{~min}$, rest, further centrifugation for $20 \mathrm{~min}$

\section{Relative infectivity}

titre

$0 \cdot 89$

$0 \cdot 10^{*}$

$1 \cdot 00$

0.95

$1 \cdot 55$

* 0.099 \pm 0.024 S.D., mean of four experiments.

\section{Effect of inoculation of monolayers during centrifuging}

To show that the refractory and unproductive binding states developed independently of the presence of organisms, we devised the technique of 'delayed inoculation' whereby organisms could be introduced to monolayers which were already being centrifuged. After 20 min centrifugation, cell monolayers (presumed to be in the refractory state) received inoculum which was allowed to remain for $40 \mathrm{~min}$. There was a 10 -fold reduction in infection resulting from entry of cells into the refractory and, subsequently, the unproductive binding state (Table $3 a$ and $b$ ). Additional experiments (Table $3 c$ and $d$ ) indicated that the induced states were reversible. Monolayers brought to rest after $60 \mathrm{~min}$ centrifugation and then recentrifuged showed the same susceptibility to infection as virgin monolayers. It thus became possible to demonstrate both reversibility of the cell states appearing during centrifugation and lack of heterogeneity among the organism or cell population which could have resulted in preferential interaction during productive binding. Organisms unattached after $20 \mathrm{~min}$ centrifugation were capable of productive binding to residual cells when monolayers were brought to rest and then, without removing the supernatants, recentrifuged for $20 \mathrm{~min}$ (Table $3 e$ ). It appeared, therefore, that organisms remained in supernatants after the first 20 min centrifugation not because they or the cell population were inherently incapable of interaction but because the rate of cell change to the refractory state was greater than the rate of organism attachment.

\section{DISCUSSION}

How centrifugation affects cell surface structures is not known. The compressive effects of pressure and directional force exerted by centrifuging may induce their movement. Equally, sedimentation of intracellular structures might indirectly affect membrane receptors via cytoskeletal linkages. The successive, transient nature of the changes is suggestive of an elastic response which might recur during prolonged centrifugation.

The nature of the organism attachment mediated by centrifugation is unknown. Sialic acid-containing receptors have been reported for $C$. trachomatis in spontaneous infection of cell monolayers (Kuo et al., 1973). These workers attributed the generally poor attachment of organisms to a weak repulsive negative charge on the cell surface which could be neutralized by DEAE-dextran. Centrifugation, by inducing cell surface changes, may have an equivalent, if more powerful, effect. It may, however, generate a wholly non-specific interaction, as suggested by its ability to overcome antibody neutralization of receptormediated spontaneous infection. 
The phenomenon of unproductive binding and its conversion to productive infection is puzzling, whatever the mode of attachment. A possible explanation is provided by the 'zipper' hypothesis which Griffin et al. (1975) put forward to account for particle attachment, without uptake, by 'professional' phagocytes. In their model, attached particles are ingested only if sequential, circumferential interactions between particle and plasma membrane can occur over the whole particle surface. Thus, unproductively bound chlamydiae would be irreversibly attached but not ingested because of incomplete circumferential binding through lack of 'receptors' in their vicinity. The inability of rest and recentrifugation of monolayers to cause entry of organisms, given the inherent reversibility of the induced states, indicates that attached organisms must in some way constrain surface structures so that a productive binding state cannot be restored. The cold shock effect (which does not release attached organisms) implies a role for cellular components in the constraint - possibly cytoskeletal structures in view of their influence on receptor movement (Nicolson, 1976). Microtubules appear especially sensitive to cold shock (Olmsted, 1977). If these control surface components in the unproductive binding state, then their depolymerization could permit resumption of the productive binding state on recentrifugation, without the need for organism detachment.

The transient cell states that we have demonstrated during infection by the GP-IC strain of $C$. psittaci may not necessarily affect infection by other organisms. However, preliminary studies with a strain of $C$. trachomatis, the more important species in human disease, show behaviour similar to GP-IC (unpublished). Conventional application of the centrifugation technique in clinical isolation work may therefore significantly underestimate the organisms present. Perhaps of greater concern is the increasing use of centrifugation to explore organism-cell interactions in vitro as models for infection in vivo. Here it will be important to assess, in future work, how far centrifugation distorts the mechanisms which, in spontaneous infection, determine attachment, ingestion and the intracellular fate of organisms in animal hosts.

We thank Dr S. P. Spragg and other colleagues for helpful discussion. I.A. was in receipt of an MRC research studentship.

\section{REFERENCES}

Ainsworth, S., Allan, I. \& Pearce, J. H. (1976). Neutralisation and adherence of chlamydiae to mucous. surfaces. Proceedings of the Society for General Microbiology 4, 14-15.

Allan, I. \& Pearce, J. H. (1977). Serum modulation of cell susceptibility to chlamydial infection. FEMS Microbiology Letters 1, 211214.

Allan, I., Spragg, S. P. \& Pearce, J. H. (1977). A pressure component in centrifuge-assisted chlamydial infection of cell cultures. FEMS Microbiology Letters 2, 79-82.

Bryden, A. S., Davies, H. A., Thouless, M. E. \& FlewetT, T. H. (1977). Diagnosis of rotavirus infection by cell culture. Journal of Medical Microbiology 10, 121-125.

Griffin, F. M., Griffin, J. A., Leider, J. E. \& Silverstein, S. C. (1975). Studies on the mechanism of phagocytosis. I. Requirements for circumferential attachment of particle-bound ligands to specific receptors on the macrophage plasma membrane. Journal of Experimental Medicine 142, 1263-1282.

Griffiths, M. S., Ainsworth, S. \& Pearce, J. H. (1976). Infectivity titration of guinea pig inclusion conjunctivitis agent in irradiated McCoy cells. Journal of General Microbiology 95, 249-256.
Howard, L., Orenstein, N. S. \& King, N. W. (1974). Purification on renografin density gradients of Chlamydia trachomatis grown in the yolk sac of eggs. Applied Microbiology 27, 102-106.

Kuo, C. C., WANG, S. P. \& Grayston, J. T. (1973). Effect of polycations, polyanions, and neuraminidase on the infectivity of trachoma-inclusion conjunctivitis and lymphogranuloma venereum organisms in HeLa cells: sialic acid residues as possible receptors for trachoma-inclusion conjunctivitis. Infection and Immunity 8, 74-79.

NicolsoN, G. L. (1976). Transmembrane control of the receptors on normal and tumor cells. I. Cytoplasmic influence over cell surface components. Biochimica et biophysica acta 457, 57-108.

Olmsted, J. B. (1977). Microtubules and flagella. In International Cell Biology 1976-1977, pp. 343347. Edited by B. R. Brinkley \& K. R. Porter. New York: The Rockefeller University Press.

OsBorN, J. E. \& WALKER, D. L. (1968). Enhancement of infectivity of murine cytomegalovirus in vitro by centrifugal inoculation. Journal of Virology 2, 853-858.

Silverstein, S. C., Steinman, R. M. \& Cohn, Z. A. (1977). Endocytosis. Annual Review of Biochemistry 46, 669-722. 